\title{
Impacto de la introducción de la vacuna contra el rotavirus en la hospitalización por gastroenteritis aguda grave en el Hospital del Niño de la Ciudad de Panamá
}

\author{
Javier Nieto Guevara, ${ }^{1}$ Óscar López ${ }^{1}$ y Gala González ${ }^{1}$
}

Forma de citar Nieto Guevara J, López O, González G. Impacto de la introducción de la vacuna contra el rotavirus en la hospitalización por gastroenteritis aguda grave en el Hospital del Niño de la Ciudad de Panamá. Rev Panam Salud Publica. 2008;24(3):189-94.

RESUMEN Objetivos. Determinar si la introducción de la vacunación infantil contra el rotavirus en Panamá permitió reducir la tasa de hospitalización por gastroenteritis en niños menores de 5 años. Métodos. Estudio observacional de corte transversal en dos períodos: del 1 de enero al 31 de agosto de 2005 (antes de la introducción de la vacunación contra el rotavirus) y del 1 de enero al 31 de agosto de 2007 (un año después de la introducción). Se estudiaron todos los niños y niñas entre 2 meses y 5 años de edad hospitalizados con diagnóstico de gastroenteritis aguda grave en la sala de corta estancia de gastroenteritis del Hospital del Niño, en Ciudad de Panamá, Panamá. Las variables fueron: número de episodios de gastroenteritis, número de casos hospitalizados por gastroenteritis aguda grave, días de hospitalización y uso de antibióticos, según dos grupos de edad (de 2 meses a 1 año y de más de 1 año a 5 años). Se calculó el riesgo relativo (RR) con intervalos de confianza de 95\% (IC95\%) y un nivel de significación $\mathrm{P} \leq 0,05$.

Resultados. Se contabilizaron 1240 episodios de gastroenteritis aguda grave en 1222 niños. No se encontraron diferencias significativas entre los dos períodos de estudio en cuanto al número de complicaciones $(\mathrm{P}=0,92)$ y defunciones $(\mathrm{P}=1,00)$. Hubo más episodios de gastroenteritis aguda grave después de la introducción de la vacuna contra el rotavirus humano que en el período previo, pero esta diferencia no fue estadísticamente significativa $(R R=1,12$; IC95\%: 0,87 a 1,44; $P=0,39$ ). No se encontraron diferencias significativas en relación al tiempo de hospitalización por grupos de edad en los periodos analizados. La proporción de casos tratados con antibióticos fue similar en ambos períodos de estudio $(29,7 \%$ contra $25,2 \% ; \mathrm{P}=0,08)$.

Conclusiones. No se encontró que la introducción de la vacunación infantil contra el rotavirus en Panamá llevara a una reducción significativa en la tasa de hospitalización por gastroenteritis en niños menores de 5 años. No se observaron cambios significativos en la morbimortalidad y el uso de antibióticos en el tratamiento de la gastroenteritis aguda grave después de la introducción de la vacuna.

Palabras clave Vacuna contra rotavirus, diarrea infantil, Panamá.

1 Departamento de Infectología, Hospital del Niño, Ciudad de Panamá, Panamá. La correspondencia se debe dirigir a Javier Nieto Guevara, Departamento de Infectología, Hospital del Niño, Dorado Lakes, Calle F Casa 73 Modelo LakeSide, Ciudad de Panamá 0830-00036, Panamá. Correo electrónico: nietdom@cableonda.net
Cada año, el rotavirus causa $111 \mathrm{mi}$ llones de casos de gastroenteritis que requieren cuidados en casa, 25 millones de visitas médicas, 2 millones de hospitalizaciones $\mathrm{y}$ entre $352000 \mathrm{y}$
592000 muertes, de ellas alrededor de 440000 en niños menores de 5 años de edad (1). La infección por rotavirus es la segunda causa de muerte en el mundo en este grupo de edad, solo su- 
perada por las infecciones respiratorias (2). Se estima que al cumplir 5 años, un niño promedio ha tenido al menos un episodio de gastroenteritis por rotavirus, uno de cada 5 niños ha visitado una unidad de salud y uno de cada 65 ha estado hospitalizado por esa causa. Aproximadamente uno de cada 293 niños muere por rotavirosis antes de cumplir 5 años (1), alrededor de $82 \%$ de ellos en los países en desarrollo.

La incidencia de la infección por rotavirus en niños es similar en todo el mundo, independientemente del nivel de desarrollo de los países, ya que este patógeno se transmite de persona a persona y es difícil de controlar mediante mejoras higiénicas. No obstante, la mortalidad es mayor en niños de países en vías de desarrollo debido al menor acceso a los medios de rehidratación y la elevada prevalencia de desnutrición (1).

El pico en la incidencia de la diarrea por rotavirus ocurre entre los 6 y 24 meses de edad, sin embargo, en países en vías de desarrollo la infección puede aparecer en lactantes menores de 6 meses (3). La mayoría de los casos de enfermedad por rotavirus se deben a los serotipos G1, G2, G3 y G4, no obstante el serotipo G5 desempeña un papel epidemiológico cada vez más importante en algunos países, como Brasil (3). Según datos de Argentina, Brasil, Chile, Costa Rica, Ecuador, Honduras, México, Panamá y Venezuela, la proporción de niños con gastroenteritis por rotavirus tratados en hospitales varía de 11,4\% a 60,0\% y más de la mitad de las hospitalizaciones se deben al serotipo G1 (4).

En Panamá se calcula que alrededor de $15 \%$ de los lactantes de 6 meses y hasta $85 \%$ de los niños de 1 año de edad han tenido al menos un episodio de infección por rotavirus (5). Según publicaciones recientes, la transmisión de esta infección en Panamá ocurre fundamentalmente entre los meses de enero a abril, con tasas de incidencia que fluctúan entre $4 \%$ y $67 \%$ (5). El tiempo promedio de hospitalización es de al menos 48 horas y los padres pierden alrededor de 10 horas de pago laboral por atender a sus hijos hospitalizados $(4,5)$.
La aplicación de la vacuna Rota Shield ${ }^{\circledR}$, aprobada para su uso en los Estados Unidos de América e introducida en el programa de vacunación de ese país en 1998, produjo una complicación conocida como invaginación intestinal que llevó a retirar este producto del mercado. Esto pudo deberse al elevado inóculo viral y a la predisposición de algunos niños a esta complicación después de la exposición a ciertas cepas $(6,7)$. La incidencia de la invaginación intestinal puede variar entre 18 y 89 casos por 100000 niños menores de 1 año (8).

Dos nuevas vacunas contra el rotavirus se desarrollaron posteriormente. Una de ellas, Rotateq ${ }^{\circledR}$ (Merck Research Laboratories, New Jersey, Estados Unidos), recibió la autorización para su aplicación en los Estados Unidos en febrero de 2006. Esta vacuna viva atenuada está compuesta por cinco cepas reagrupadas a partir de aislamientos en humanos y bovinos. Cuatro de ellas expresan las proteínas exteriores G1, G2, G3 y G4 de la cápside viral de los rotavirus parentales humanos correspondientes y la proteína de fijación VP7 del rotavirus parental bovino. El quinto virus reagrupado expresa la proteína de fusión P1AP[8] del rotavirus parental humano y la proteína G6 de la cápside viral del rotavirus parental bovino. Según los resultados de los estudios clínicos, la vacuna oral Rotateq $^{\circledR}$ ha mostrado una buena tolerancia y provee una adecuada protección contra la enfermedad por rotavirus, ya que induce la producción de anticuerpos neutralizantes de los serotipos humanos más frecuentes (9). En un estudio multicéntrico realizado en 11 países no se observaron casos de invaginación intestinal ni otros eventos adversos graves (9).

La segunda vacuna aprobada, Rotarix $^{\circledR}$ (GlaxoSmithKline Biologicals, Bélgica), es una vacuna oral monovalente de virus vivo atenuado con una cepa G1P[8] que expresa las proteínas VP4 y VP7 de la mayoría de los serotipos de rotavirus humanos. En el ensayo multicéntrico Rota-023 se evaluó la eficacia de esta vacuna en una muestra de 63225 infantes de 2 a 4 meses de edad de 11 países latinoamericanos y Finlandia (10). La vacuna demostró una eficacia de $85 \%$ en la prevención de la gastroenteritis aguda grave, mientras que la gastroenteritis por cualquier causa se redujo en $43 \%$ (10).

Según estudios de la relación costobeneficio realizados en 4025 lactantes en Panamá, la introducción de la vacuna antirrotavírica podría evitar anualmente 13685 visitas médicas ambulatorias, 1140 hospitalizaciones y 24 muertes, en los primeros 5 años de vida (11). En consecuencia, el gobierno panameño decidió incorporar la vacuna antirrotavírica Rotarix $^{\circledR}$ al Programa Ampliado de Inmunizaciones el 14 de marzo de 2006 y vacunar a los 70000 niños que nacen como promedio anualmente en ese país.

El objetivo de la presente investigación fue determinar si la introducción de la vacunación infantil contra el rotavirus en Panamá permitió reducir la tasa de hospitalización por gastroenteritis en niños menores de 5 años.

\section{MATERIALES Y MÉTODOS}

Se realizó un estudio observacional de corte transversal en los períodos comprendidos entre el 1 de enero y el 31 de agosto de 2005 y entre el 1 de enero y el 31 de agosto de 2007. En el primer período de estudio se captaron todos los niños y niñas entre 2 meses y 5 años de edad hospitalizados con diagnóstico de gastroenteritis aguda grave en la sala de corta estancia de gastroenteritis del Hospital del Niño, en Ciudad de Panamá, Panamá. La captación de los participantes se realizó a partir de la base de datos de hospitalizaciones, provista por el Servicio de Estadísticas y Archivos Clínicos del Hospital del Niño. Se completó un modelo de recogida de datos con el número de registro del niño, el distrito de procedencia, su edad, tiempo de hospitalización, complicaciones y uso de antibióticos.

Durante el segundo período de estudio se utilizaron los mismos criterios de selección, pero se estudiaron solamente los niños en cuyos expedientes clínicos aparecía la anotación de que 
habían recibido las dosis necesarias de la vacuna antirrotavírica para su edad. Según las regulaciones vigentes, la vacuna Rotarix ${ }^{\circledR}$ se aplicó en dos dosis: a los 2 y a los 4 meses de edad, con un intervalo de 8 semanas; los niños entre 5,5 y 6 meses de nacido recibían una sola dosis. De esta manera, todos los niños participantes en la segunda etapa (2007) debían haber recibido al menos una dosis de la vacuna, aunque podían no haber recibido el esquema de vacunación completo. La información fue transcrita y analizada en una base de datos del programa estadístico SPSS versión 13.0.

La cobertura de vacunación en los distritos de Panamá, Arraiján, San Miguelito, La Chorrera, Chepo, Capira, Balboa, Chame y San Carlos para la primera y segunda dosis fue de $97,2 \%$ y $69,1 \%$, respectivamente, mientras que para las regiones del interior de la república y las comarcas indígenas fue de $76,2 \%$ y $48,3 \%$, para cada una de las dosis respectivamente.

La gastroenteritis aguda grave se definió como la enfermedad con diarrea (tres o más deposiciones líquidas en un período de 24 horas), acompañada de vómitos o sin ellos, que requirió la hospitalización en una institución médica con tratamiento equivalente al plan $C$ de la Organización Mundial de la Salud (12). Se excluyeron los niños que presentaron heces mucosanguinolentas o sanguinolentas, con cultivo de heces positivo para enterobacterias patógenas o diagnóstico de enfermedad parasitaria, así como los que presentaban otra enfermedad crónica gastrointestinal concomitante, inmunodeficiencia o que desarrollaron gastroenteritis aguda grave en las 72 horas posteriores a su hospitalización.

Las variables de resultado fueron el número de episodios de gastroenteritis, el número de casos hospitalizados por gastroenteritis aguda grave, los días de hospitalización y el uso de antibióticos, según dos grupos de edad: de 2 meses a 1 año y de más de 1 a 5 años. Los resultados se expresaron en tasas, números absolutos, frecuencias relativas y porcentajes y se utilizaron las pruebas de la $t$ de Student y de la ji al cuadrado, según el tipo de variable. Se calculó el riesgo relativo (RR) con intervalos de confianza de 95\% (IC95\%). Se empleó un nivel de significación $P \leq 0,05$.

\section{RESULTADOS}

En total se identificaron 1248 niños con gastroenteritis aguda grave, de ellos se excluyeron 26 (14 por presentar gastroenteritis aguda grave en las 72 horas siguientes a su hospitalización, 11 por desnutrición grave que podía afectar a su capacidad inmunitaria y 1 por invaginación intestinal). Los 1222 restantes (405 niños de 2 meses a 1 año y 817 de más de 1 año a 5 años) presentaron 1240 episodios de gastroenteritis aguda grave, para una tasa de 1,02 episodios por niño. En el cuadro 1 se muestran las características generales de los participantes en el estudio. No se encontraron diferencias estadísticamente significativas entre los niños analizados en cada uno de los dos períodos.

El mayor número de hospitalizaciones por gastroenteritis aguda grave en ambos períodos de estudio provinieron de los distritos de Panamá, Arraiján y San Miguelito (figura 1).

No se encontraron diferencias significativas entre los dos períodos de estudio en cuanto al número de complicaciones $(P=0,92)$ y defunciones $(P=1,00)$ (cuadro 2). Hubo más episodios de gastroenteritis aguda grave - en gene- ral y en los dos grupos de edad- en el período posterior a la introducción de la vacuna contra el rotavirus humano (2007) que en el período previo (2005), no obstante, estas diferencias no fueron estadísticamente significativas $(\mathrm{RR}=1,12$; IC95\%: 0,87 a 1,44 ; $P=0,39)$. Esta relación se reflejó en las tasas de hospitalización por gastroenteritis aguda grave por 1000 hospitalizaciones en ambos períodos (figura 2).

Tanto en 2005 como en 2007, las causas más frecuentes de complicación y muerte fueron la convulsión febril y la sepsis, respectivamente (datos no mostrados).

La proporción de casos tratados con antibióticos fue similar en ambos períodos de estudio $(29,7 \%$ contra $25,2 \%$; $P=0,08)$. No hubo diferencia estadísticamente significativa en cuanto al tiempo de hospitalización, tanto en el grupo de menores de 1 año como en los niños de más de 1 año (figura 3).

\section{DISCUSIÓN}

En este estudio se evaluó el impacto de la vacuna contra el rotavirus humano sobre la incidencia de la gastroenteritis aguda grave en una instalación de salud de tercer nivel de la Ciudad de Panamá.

No se encontró un efecto significativo de la vacunación en la reducción del número de hospitalizaciones tota-

CUADRO 1. Características generales de los niños hospitalizados con gastroenteritis aguda grave en los períodos estudiados, Hospital del Niño, Ciudad de Panamá, Panamá

\begin{tabular}{|c|c|c|c|c|c|}
\hline \multirow[b]{2}{*}{ Característica } & \multicolumn{2}{|c|}{2005} & \multicolumn{2}{|c|}{2007} & \multirow[b]{2}{*}{$P^{a}$} \\
\hline & No. & $\%$ & No. & $\%$ & \\
\hline \multicolumn{6}{|l|}{ Sexo } \\
\hline Niños & 261 & 55,3 & 417 & 55,6 & \multirow{2}{*}{0,96} \\
\hline Niñas & 211 & 44,7 & 333 & 44,4 & \\
\hline \multicolumn{6}{|l|}{ Edad $^{\mathrm{b}}$} \\
\hline De 2 meses a 1 año & 168 & 35,6 & 237 & 31,6 & \multirow{2}{*}{0,17} \\
\hline De más de 1 año a 5 años & 304 & 64,4 & 513 & 68,4 & \\
\hline \multicolumn{6}{|l|}{ Distrito de procedencia } \\
\hline Panamá & 446 & 94,5 & 716 & 95,5 & \multirow{3}{*}{0,52} \\
\hline Otros & 26 & 5,5 & 34 & 4,5 & \\
\hline Total & 472 & 100,0 & 750 & 100,0 & \\
\hline
\end{tabular}


les, complicaciones, muertes o casos tratados con antibióticos durante la hospitalización. Diversos factores pueden haber influido en estos resultados. En primer lugar, según cifras del Programa Ampliado de Inmunizaciones del Ministerio de Salud de Panamá, la cobertura de la primera dosis durante el primer semestre de 2007 fue de $66,5 \%$, mientras que para la segunda dosis fue solamente de $57,9 \%$, por lo que una gran parte de la cohorte anual de nacidos vivos no quedó protegida. En segundo lugar, en 30\% de los expedientes clínicos se encontró la anota- ción "vacunación completa para la edad", sin especificarse el número de dosis recibidas, por lo que se desconoce el número real de niños que recibieron el esquema completo de inmunización. En tercer lugar, la posible reducción en el número de episodios de gastroenteritis aguda grave por rotavirus después de la vacunación puede haber quedado enmascarada por la circulación de otros patógenos virales no diagnosticados. Por último, algunos factores socioeconómicos no controlados relacionados con deficiencias en el saneamiento ambiental y las

FIGURA 1. Número de casos de gastroenteritis aguda grave hospitalizados, según el distrito de procedencia del paciente en los períodos estudiados, Hospital del Niño, Ciudad de Panamá, Panamá

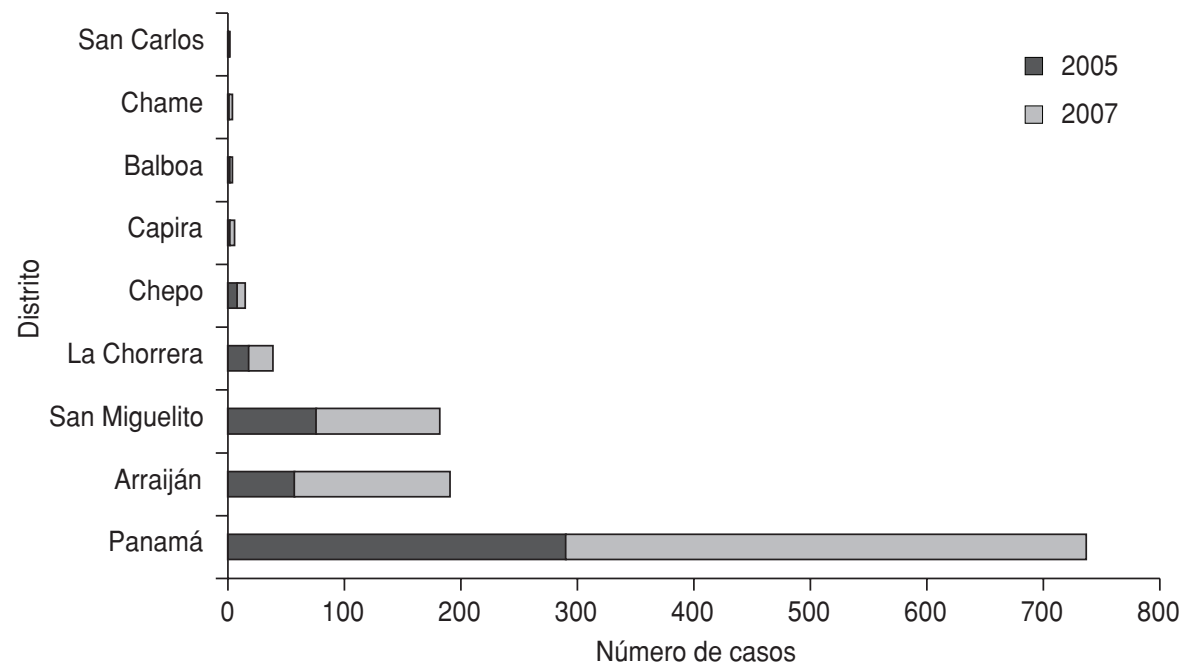

Fuente: Expedientes clínicos del Hospital del Niño, Ciudad de Panamá, Panamá.

condiciones de hacinamiento pudieron haber variado en ese lapso e influido en estos resultados; la mayoría de los casos procedían de zonas de alta densidad poblacional.

La participación en el segundo período de niños que recibieron al menos una dosis de la vacuna no debe haber alterado de forma significativa los resultados de este trabajo. Según Velázquez y colaboradores (13), la infección por rotavirus produce anticuerpos que permanecen en el organismo y contribuyen a reducir la gravedad de los episodios diarreicos agudos provocados por exposiciones subsecuentes a este virus. Algunos de los infantes estudiados pudieron haber tenido una infección previa por rotavirus, además de la vacunación - ya sea con una o dos dosis-, lo que pudo aumentar el estado de inmunización.

La baja cobertura de la vacunación con la segunda dosis en muchos distritos y la incidencia de otros factores ajenos a la vacuna - como una más rápida y adecuada hidratación de los pacientes o la circulación de agentes virales de menor virulencia- pudieron llevar a la reducción del tiempo de hospitalización, aunque esta diferencia no fue estadísticamente significativa. Es importante anotar el hecho de que no se documentó una reducción significativa en el uso de antibióticos después de la introducción de la vacuna contra el rotavirus humano con respecto al período de estudio previo a la vacunación. El uso continuado e indiscriminado de anti-

CUADRO 2. Principales indicadores en los casos hospitalizados por gastroenteritis aguda grave en los períodos estudiados, Hospital del Niño, Ciudad de Panamá, Panamá

\begin{tabular}{|c|c|c|c|c|c|c|c|}
\hline \multirow[b]{2}{*}{ Indicador } & \multicolumn{2}{|c|}{2005} & \multicolumn{2}{|c|}{2007} & \multirow{2}{*}{$\begin{array}{l}\text { Riesgo } \\
\text { relativo }\end{array}$} & \multirow[b]{2}{*}{$1 \mathrm{C} 95 \% \mathrm{a}$} & \multirow[b]{2}{*}{$P^{\mathrm{b}}$} \\
\hline & No. & $\%$ & No. & $\%$ & & & \\
\hline Hospitalizaciones & 481 & 100,0 & 759 & 100,0 & 1,12 & $0,87-1,44$ & 0,39 \\
\hline Menores de 1 año & 168 & 34,9 & 246 & 32,4 & 1,07 & $0,93-1,24$ & 0,39 \\
\hline De más de 1 año a 5 años & 313 & 65,1 & 513 & 67,6 & & & \\
\hline Complicaciones & 30 & 6,2 & 47 & 6,2 & 1,01 & $0,75-1,34$ & 0,92 \\
\hline Defunciones & 1 & 0,2 & 1 & 0,1 & 1,29 & $0,32-5,17$ & 1,00 \\
\hline Tratamiento con antibióticos & 143 & 29,7 & 191 & 25,2 & 1,15 & $0,99-1,33$ & 0,08 \\
\hline
\end{tabular}

Fuente: Expedientes clínicos del Hospital del Niño, Ciudad de Panamá, Panamá.

a IC95\%: intervalo de confianza de 95\%.

b Prueba de la ji al cuadrado. Nivel de significación de $P<0,05$ 
FIGURA 2. Tasas de hospitalización por gastroenteritis aguda grave en los períodos estudiados, Hospital del Niño, Ciudad de Panamá, Panamá

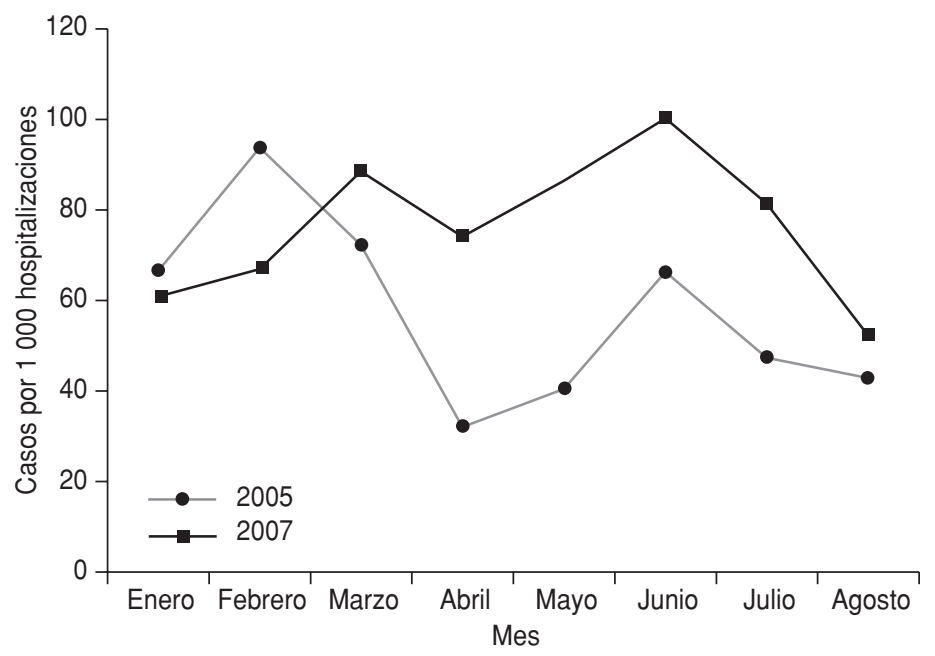

Fuente: Expedientes clínicos del Hospital del Niño, Ciudad de Panamá, Panamá.

FIGURA 3. Tiempo de estancia hospitalaria e intervalos de confianza de $95 \%$ por grupo de edad en los períodos estudiados, Hospital del Niño, Ciudad de Panamá, Panamá

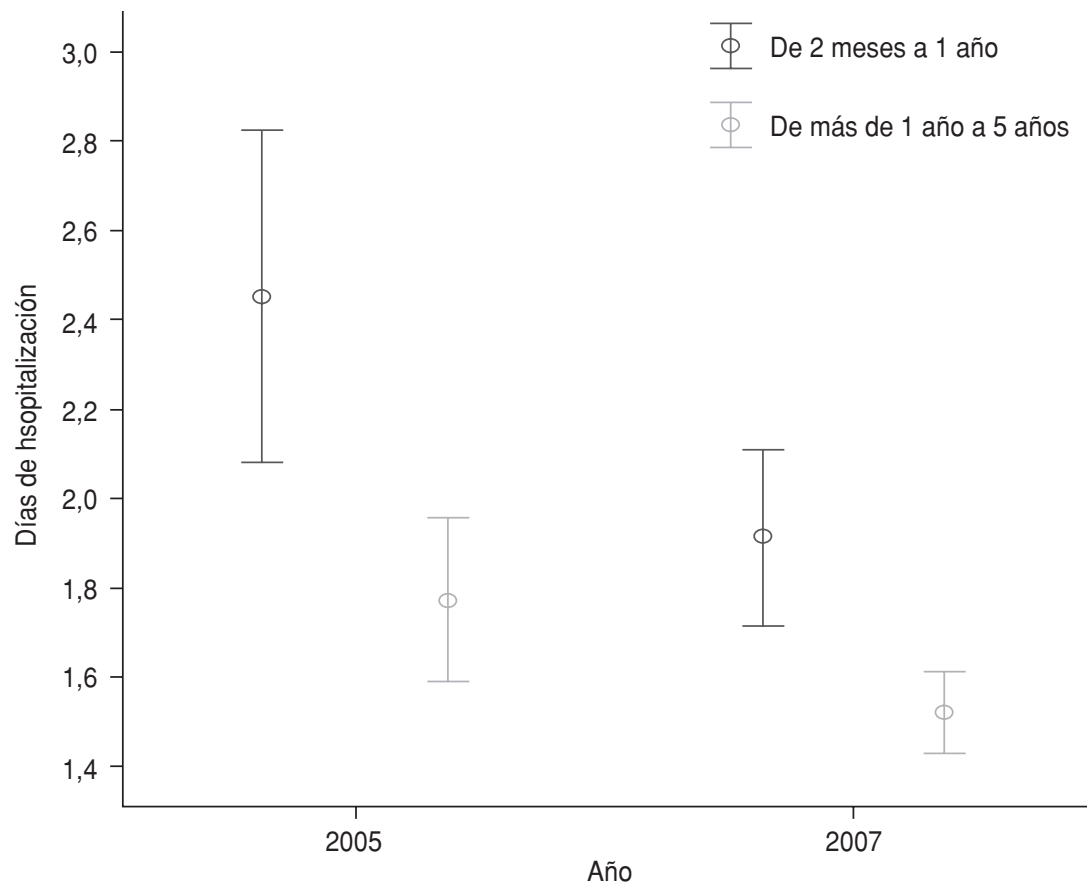

Fuente: Expedientes clínicos del Hospital del Niño, Ciudad de Panamá, Panamá.

bióticos para tratar las enfermedades diarreicas agudas puede elevar el riesgo de emergencia de microorganismos resistentes y los costos totales directos (4).
La convulsión febril - la complicación más frecuente en ambos períodos de estudio - se ha asociado con los casos de influenza, adenovirus, parainfluenza y, en menor proporción, a la infección por el virus sincitial respiratorio y el rotavirus (14). La baja mortalidad por gastroenteritis aguda grave encontrada en ambos períodos de estudio, a diferencia de la publicada en otros países $(15,16)$, contrasta con el número elevado de casos de hospitalización por gastroenteritis aguda grave en los períodos analizados, por lo que una estrategia de intervención adecuada podría enfocarse en reducir el número de hospitalizaciones por gastroenteritis aguda grave.

Dada la naturaleza observacional de este estudio, basado en la recolección retrospectiva de información, la mala calidad y la falta de datos en los expedientes clínicos pueden haber influido negativamente en la validez interna de los resultados.

En conclusión, no se encontró que la introducción de la vacunación infantil contra el rotavirus en Panamá llevara a una reducción significativa en la tasa de hospitalización por gastroenteritis en niños menores de 5 años. No se observaron cambios significativos en la morbimortalidad y el uso de antibióticos en el tratamiento de la gastroenteritis aguda grave después de la introducción de la vacuna.

Se recomienda mejorar los porcentajes de cobertura de la vacunación contra el rotavirus, sobre todo durante la aplicación de la segunda dosis en el distrito de Panamá y muy especialmente en el interior del país y las comarcas indígenas. Es necesario mejorar la calidad de la información de los expedientes clínicos con respecto al cumplimiento de los programas de vacunación. Una vez resueltas estas deficiencias, se deben realizar estudios de casos y controles con variables controladas que permitan evaluar el impacto real de la vacunación contra el rotavirus y emprender estudios prospectivos que tengan una mayor validez interna.

Agradecimientos. Al Departamento de Archivos Clínicos y Estadísticas del Hospital del Niño de Ciudad de Panamá, Panamá, por su valiosa ayuda, sin la cual no hubiera sido posible esta investigación. 


\section{REFERENCIAS}

1. Parashar UD, Hummelman EG, Bresse JS, Miller MA, Glass RI. Global illness and deaths caused by rotavirus disease in children. Emerg Infect Dis. 2003;9(5):565-72.

2. World Health Organization. Annual number of deaths by cause for children under five years of age in WHO regions, estimates for 2000-2003. En: WHO. World Health Report 2005. Geneva: WHO; 2005. Hallado en http:// www.who.int/whr/2005/annex/annexes 3-4 en.pdf. Acceso el 9 de junio de 2008.

3. Linhares AC, Bresee JS. Rotavirus vaccines and vaccination in Latin America. Pan Am J Public Health. 2000;8(5):305-30.

4. Rheingans RD, Constenla D, Antil L, Innis BL, Breuer T. Potential cost-effectiveness of vaccination for rotavirus gastroenteritis in eight Latin American and Caribbean countries. Emerg Infect Dis. 2006;12(2):304-6.

5. Rheingans RD, Constenla D, Antil L, Innis BL, Breuer T. Economic and health burden of rotavirus gastroenteritis for the 2003 birth cohort in eight Latin American and Caribbean countries. Rev Panam Salud Publica. 2007;21(4):192-204.
6. Nakagomi T. Rotavirus infection and intussusception: a view from retrospect. Microbiol Immunol. 2000;44(8):619-28.

7. Bines J. Intussusception and rotavirus vaccines. Vaccine. 2006;24(18):3772-6.

8. Sáez-Llorens X, Guevara JN. Intussusception and rotavirus vaccines: what is the background risk? Pediatr Infect Dis J. 2004;23(4): 363-5.

9. Vesikari T, Matson DO, Dennehy P, Van Damme P, Santosham M, Rodríguez Z, et al. Safety and efficacy of a pentavalent humanbovine (WC3) reassortant rotavirus vaccine. N Engl J Med. 2006;354:23-33.

10. Ruiz-Palacios GM, Pérez-Schael I, Velázquez FR, Abate H, Breuer T, Costa Clemens SA, et al. Safety and efficacy of an attenuated vaccine against severe rotavirus gastroenteritis. N Engl J Med. 2006;354(1):11-22.

11. Constenla D, Ortega-Barría E, Rheingans RD, Antil L, Sáez-Llorens X. Impacto económico de la vacuna antirrotavirus en Panamá. An Pediatr. 2008;68(2):128-35.
12. Organización Panamericana de la Salud. Manual de tratamiento de la diarrea. Washington, D.C: OPS; 1987. (Serie Paltex No. 13).

13. Velázquez FR, Matson DO, Calva JJ, Guerrero $\mathrm{ML}$, Morrow AL, Carter-Campbell S, et al. Rotavirus infection in infants as protection against subsequent infections. N Engl J Med. 1996;335:1022-8.

14. Chung B, Wong V. Relationship between five common viruses and febrile seizure in children. Arch Dis Child. 2007;92(7):589-93.

15. Glass RI, Bresee JS, Turcios R, Fischer TK, Parashar UD, Steele AD. Rotavirus vaccines: targeting the developing world. J Infect Dis 2005;192(supl1):S160-6.

16. Parashar UD, Gibson CJ, Bresse JS, Glass RI. Rotavirus and severe childhood diarrhea. Emerg Infect Dis. 2006;12(2):304-6.

Manuscrito recibido el 27 de octubre de 2007. Aceptado para publicación, tras revisión, el 16 de abril de 2008.

ABSTRACT Objectives. To determine if infant rotavirus vaccination in Panama has reduced the rate of hospital admission for gastroenteritis among children under 5 years of age.

Methods. An observational, cross-sectional study of two time periods: 1 January-

Impact of rotavirus vaccine introduction on hospital admissions for severe acute gastroenteritis at the Children's Hospital in Panama City 31 August 2005 (prior to initiating rotavirus vaccination) and 1 January-31 August 2007 (one year after introducing rotavirus vaccination). All the children from 2 months -5 years of age admitted with a diagnosis of acute gastroenteritis to the shortstay gastroenteritis area of the Children's Hospital in Panama City, Panama, were studied. The variables were: number of gastroenteritis episodes; number of cases admitted for severe acute gastroenteritis; number of days hospitalized; and antibiotics treatment for each of the two age groups ( 2 months -1 year and $>1-5$ years). The relative risk (RR) was calculated with a $95 \%$ confidence interval $(95 \% \mathrm{CI})$ and a significance level of $P \leq 0.05$.

Results. There was a total of 1240 episodes of severe acute gastroenteritis in 1222 children. No significant differences were found between the two study periods regarding the number of complications $(P=0.92)$ and deaths $(P=1.00)$. Although there were more episodes of severe acute gastroenteritis after initiating vaccination against human rotavirus than there were in the period prior, the difference was not statistically significant ( $\mathrm{RR}=1.12 ; 95 \% \mathrm{CI}$ : $087-1.44 ; P=0.39)$. There were no significant differences found in the length of hospital stay by age groups studied in each time period. The percent of cases treated with antibiotics was similar in both study periods ( $29.7 \%$ versus $25.2 \% ; P=0.08$ ).

Conclusions. The introduction of infant rotavirus vaccination was not found to lead to a significant reduction in hospital admission rates for gastroenteritis among children less than 5 years of age. Significant changes in morbidity and in antibiotics use were not found after the introduction of the vaccine.

Key words Rotavirus vaccines; diarrhea, infantile; Panama. 